\title{
Safety and Security of Menopausal Therapies
}

\author{
a report by \\ Tommaso Simoncini, MD, PhD, ${ }^{1}$ Chiara Baldacci, MD, ${ }^{1}$ and Andrea Riccardo Genazzani, MD, PhD ${ }^{2}$ \\ 1. Molecular \& Cellular Gynecological Endocrinology Laboratory, Department of Reproductive Medicine and Child Development, University of Pisa;
}

\author{
2. President, International Society of Gynecological Endocrinology
}

DOI: 10.17925/USE.2007.00.1.80

The climacteric syndrome is a complex condition characterized by a set of symptoms and degenerative changes that ensue due to the decline in production of sex steroids by the ovaries. Hence, there is a need for safe methods for the short- and long-term management of postmenopausal women. Hormone-replacement therapy (HRT) is widely used for the relief of menopausal symptoms and for the prevention of diseases linked to longterm hormonal deprivation. The use of HRT has increased rapidly in the past decade, but is currently a subject of debate because of the possible negative effects on the breast and on the cardiovascular system. Other molecules have been studied as alternatives to standard HRT to also relieve symptoms in women with contraindications to hormones or who refuse sex steroids for personal reasons. The aim of this article is to discuss the results of the principal studies performed in the past few years on the safety of HRT and of other climacteric therapies.

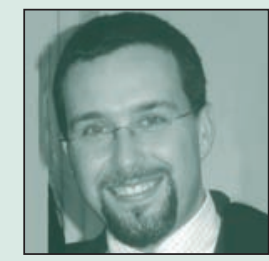

Tommaso Simoncini, MD, PhD, is Assistant Professor and Head of the Molecular \& Cellular Gynecological Endocrinology Laboratory (MCGEL) in the Department of Reproductive Medicine and Child Development at the University of Pisa, where his main research interest is the signal transduction of steroid receptors. He is also a practicing gynecologist, with interests in gynecological endocrinology, menopause, and gynecological surgery. Dr Simoncini is Secretary General of the Italian Society of Gynecological Endocrinology and the Executive Secretary of the International Society of Gynecological Endocrinology (ISGE). He has authored more than 40 papers in international journals.

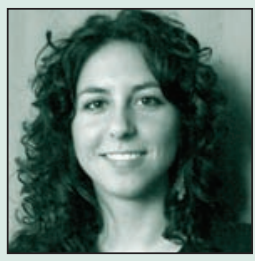

Chiara Baldacci, MD, has been undertaking research activities in the MCGEL in the Department of Reproductive Medicine and Child Development at the University of Pisa since 2004. She is the recipient of the 2005-2006 Giovanni Guelfi International Award in Biology and Biomedicine from the Accademia Nazionale dei Lincei. Dr Baldacci earned her medical degree in 2005 from the University of Pisa.

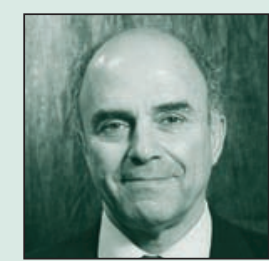

Andrea Riccardo Genazzani, MD, PhD, is President of the ISGE and President of the European Society for Gynecologic and Obstetric Investigation. He is Director of the Department of Obstetrics and Gynecology at the University of Pisa. He is Past President of the Institute of Metabolic Science (IMS) and was a member of the Scientific and Ethical Review Group of the World Health Organization Research, Development and Training in Human Reproduction program from 1998 to 2002. He is a member of various international and national scientific societies, a Fellow ad eundem of the Royal College of Obstetricians and Gynaecologists, and Editor-in-Chief of Gynecological Endocrinology. He has authored more than 537 papers in peerreviewed journals and has edited more than 42 books.
The Menopause

The climacteric is the consequence of the withdrawal of estradiol and progesterone due to the cessation of the cyclical ovarian function. This hormonal change affects a large series of bodily targets, causing atrophy of tissues and metabolic modifications along with psychological and sexual changes, which are experienced variably by women. The first signs include the typical vasomotor symptoms, nocturnal sweats, and psychological instability. Soon after these first manifestations, atrophic changes and metabolic and body composition changes develop. Changes in the lipid profile and in body-fat distribution and quantity are associated with estrogen deprivation and lead to a change in the metabolism of climacteric women toward 'male' status. The skeletal system is heavily affected in most individuals, with an accelerated bone loss that can lead to osteopenia or to overt osteoporosis. The function of the cardiovascular system is also affected by the changes in circulating sex steroids, with enhanced atherosclerotic degeneration. Circumstantial evidence indicates that the central nervous system may be affected in the long term, with an increased risk of neurodegenerative diseases such as dementia.

\section{Hormone Replacement Therapy}

Associations of estrogens with or without a progestin represent the most effective therapies for climacteric symptoms, but recent findings have opened an as yet unresolved debate on the safety of these therapies, particularly in regard to breast cancer and to cardiovascular disease (CVD).

\section{Cardiovascular System}

Several long-term observational studies suggest that HRT prevents CVD in postmenopausal women. The conclusion of all of these trials is that HRT reduces the risk of coronary heart disease by about $40 \%$. However, a large, randomized, placebo-controlled trial failed to confirm this benefit."

The potential cardioprotective actions of estrogen may depend on the levels of pre-existing CVD at the time of therapy initiation. ${ }^{2}$ For example, the Estrogen Replacement and Atherosclerosis clinical trial reported that women with pre-existing atherosclerosis receive no benefit on the progression of carotid atherosclerosis by estrogen replacement. ${ }^{3}$ Thus, while estrogen may protect against the development of atherosclerosis, it does not appear to be protective against existing atherosclerosis. Studies in women with low CVD risk concur with this. Indeed, in the Women's Health Initiative (WHI) study, those women who started HRT early after the menopause, probably having less-developed CVD, showed better cardiovascular outcomes with a trend toward protection. ${ }^{4}$ However, the vast majority of women included in the WHI trial had a severely diseased vasculature due to age and to the significant prevalence of cardiovascular risk factors (obesity, high 
cholesterol, and hypertension), and this might explain the failure of this trial in demonstrating overall cardiovascular benefits with HRT.

Bones

Osteoporosis is characterized by reduced bone mass that leads to reduced bone quality and resistance and, consequently, to an increased risk of fractures. Osteoporosis is a very common condition in postmenopausal women, causing significant morbidity and reduced quality of life.

Circulating estradiol has a protective effect on the bones, reducing skeletal remodeling through many mechanisms: reduction in activation of bone metabolic units; enhanced survival of osteoclasts; improved efficiency of gastrointestinal calcium absorption; and renal calcium conservation. ${ }^{5}$

By increasing bone-mineral density (BMD), HRT is the best (and more physiological) protective therapy against osteoporosis and fractures in postmenopausal women.

In the double-blind Postmenopausal Estrogen/Progestin Intervention trial and in the Women's Health, Osteoporosis, Progestin, Estrogen trial, women treated with estrogen or estrogen/progesterone therapy had a significant increase in BMD versus the placebo group, in which a loss of bone mass was observed. ${ }^{1,6-8}$ Prospective randomized studies confirm these results. In the WHI study there was a clear reduction in the risk of fractures among women receiving continuous combined conjugated equine estrogen plus medroxyprogesterone acetate or the estrogen therapy alone. ${ }^{1,9,10}$

\section{Cognitive Function}

Due to the widespread presence of estrogen receptors throughout the brain, estrogen effects are also widespread and affect brain structure and function and provide neuroprotection against oxidative stress via antioxidant effects. ${ }^{11}$ Moreover, estradiol in vitro promotes the breakdown of the $\beta$-amyloid precursor protein, preventing the accumulation of $\beta$-amyloid. There exists, then, a biological plausibility for the clinical hypothesis that estrogen helps to maintain cognition in women and prevents or delays the development of neurodegenerative disorders.

Observational studies in which the treatment is started in the early postmenopausal period show a decreased risk of Alzheimer's disease with treatment. ${ }^{12}$ In contrast to these results, the WHI Memory Study (WHIMS) showed nearly a doubling of risk of all-cause dementia. ${ }^{13}$ One explanation for this discrepancy is that late initiation of hormone therapy (after 65 years), as in the WHIMS, may not be effective in preventing neurodegeneration and may instead precipitate vascular dementia, whereas early use confers benefit. ${ }^{2,14}$ While the prevention of Alzheimer's disease with HRT is still to be established, it seems that the initiation of HRT in patients aged 65 or more may increase the risk of impaired cognitive function.

\section{Breast Cancer}

Breast cancer is the most common cancer in women in developed countries. Estrogens have clear proliferative activity on breast-cancer cells in vivo and in vitro, ${ }^{15}$ so it may be biologically sound that prolonged exposure to estrogens increases the risk of breast cancer. However, it is not clear if exposure to estrogens has any effect on cancer development per se. Most of the currently available evidence suggests that cancer transformation may not be related to estrogen exposure but that, once this primal event takes place, estrogens may promote tumor growth and eventually spread. On the other hand, progestins have traditionally been seen as protective against breast-cancer development despite the absence of a strong biological rationale for an anti-estrogenic effect of progestins on the breast.

Correlation between HRT use and breast-cancer risk has been studied in many epidemiological studies. A large meta-analysis published in 1997 indicated that the risk of breast cancer is raised in women using HRT and increases with increasing duration of use. ${ }^{16}$ This excess risk is reduced after HRT cessation and disappears within five years. ${ }^{16}$ Recently, the WHI study reported a $26 \%$ increase in the relative risk of breast cancer for combined estrogen-progestogen compared with a placebo. However, the parallel arm of the WHI study investigating the effect of the administration of estrogens alone showed no increase of breast cancer, with a trend toward a reduction of risk. ${ }^{17}$ This study, along with the long-term analysis of the Nurse's Health Study, in general indicates that the impact of HRT on the incidence of breast cancer is limited and associated only with very long administrations. ${ }^{18}$ In addition, recent trials call for new research to better understand the role of progestins, showing that-based on the compound — the risk of breast cancer changes. ${ }^{19}$

\section{Endometrial Cancer}

The majority of all endometrial malignancies occurs mostly in perimenopausal and early post-menopausal women. The pathogenesis is, in part, linked to prolonged and excessive exposure to endogenous or exogenous estrogens, not balanced by the cyclical production of progesterone. The risk of endometrial cancer is not clearly related to the dose but rather to the duration of unopposed estrogen exposure, as longterm administration correlates with a five-fold higher risk. ${ }^{20}$ HRT with estrogen alone increases endometrial cancer risk, whatever the type and dose of estrogen and the route of administration. ${ }^{20}$ Progesterone has a well-known anti-estrogenic effect on the endometrium. ${ }^{21}$ The addition of a progestogen to the estrogen-replacement therapy is then mandatory to avoid the risk of endometrial cancer, contrasting the stimulation of the endometrium by estrogens. To this extent, in the presence of a progestin, endometrial-cancer risk is decreased either with cyclical or continuous HRT. Cyclical regimens that include more than 10 days of progestogen exposure per month appear to provide maximum protection. ${ }^{22}$

\section{Colorectal Cancer}

Colorectal carcinoma is a leading cause of illness and death in developed countries, being the second most common cancer in women after breast cancer. ${ }^{23}$ There is strong evidence to show that the incidence of colorectal carcinoma can be significantly reduced by HRT. A meta-analysis of 18 epidemiological studies of HRT and colorectal cancer showed a $20 \%$ reduction of colon- and rectal-cancer risk in women who had ever taken HRT compared with those had never taken HRT. ${ }^{23}$ In addition, the WHI trial showed a significant reduction in colon-cancer risk in HRT users. The possible biological explanation of this reduction of risk includes the effect of sex steroids on bile-acid metabolism and direct effects on the colonic epithelium. ${ }^{24}$

Other Therapies

Other molecules have been studied as alternatives to standard replacement therapy with sex-steroid hormones.

\section{Tibolone}

Tibolone is a synthetic steroid that is rapidly converted to two metabolites 
with estrogenic activity and to a third metabolite characterized by a mixed progestogenic/androgenic activity. ${ }^{25}$ Tibolone controls hot flushes, sweating, and mood symptoms and, due to its androgenic component, is effective in improving libido. ${ }^{26-28}$

\section{Cardiovascular System}

Cardiovascular clinical outcomes from randomized controlled trials are not yet available. Surrogate end-point studies for arterial disease and venous thrombo-embolic disease are inconclusive with regard to benefit or risk. ${ }^{29}$

\section{Bones}

Randomized, controlled studies show that tibolone increases BMD and reduces risk of fracture..$^{30,31}$ These beneficial effects are seen over long-term treatments (>10 years) and in both early and late post-menopausal women, as well as in women with established osteoporosis. ${ }^{32}$

\section{Breast Cancer}

The Million Women Study reported an increased risk of breast cancer in women treated with tibolone, although this was significantly lower than that seen with combined therapies with estrogens and progestins. ${ }^{33}$ However, this study has many biases, including the likely selective prescription of tibolone to women at higher risk of breast cancer due to the assumption that this compound could be less active on the breast with respect to standard HRT. The combined analysis of randomized clinical studies on tibolone indicates no increase in risk of breast cancer development compared with placebo. Tibolone treatment is associated with a reduction of proliferation and a stimulation of apoptosis in normal breast cells, which is possibly attributable to the impact of this compound on the activity of estrogen-metabolizing breast enzymes. ${ }^{34}$ Due to these findings, the administration of tibolone is currently under evaluation for the treatment of climacteric symptoms in breast cancer patients by the Livial Intervention Following Breast Cancer: Efficacy, Recurrence, and Tolerability End-points trial. ${ }^{34}$

\section{Endometrial Cancer}

The metabolization of tibolone is tissue-selective and the conversion to the progestogenic metabolite is particularly active in the endometrium. Investigation of endometrial histology in women treated with tibolone shows no hyperplasia and a high level of atrophic endometrium, indicating no proliferative effect of this molecule. ${ }^{35,36}$

\section{Phyto-estrogens}

Phyto-estrogens are biologically active compounds found in certain plants in high concentrations. They have a chemical structure similar to that of estradiol and the ability to bind to estrogen receptors exerting variable estrogenic and anti-estrogenic effects. ${ }^{37}$ Many clinical studies have been conducted to assess the effects of phyto-estrogens on post-menopausal syndrome, but, while some studies report a modest benefit compared with placebo, others do not. ${ }^{38-45}$

\section{Cardiovascular System}

In vitro, genistein-a phyto-estrogenic molecule-stimulates the synthesis of nitric oxide from endothelial cells. ${ }^{46}$ Clinical trials demonstrate that consuming $25-50 \mathrm{~g}$ per day of soy protein is effective in reducing low-density lipoprotein cholesterol by approximately $4-8 \%$, and the Framingham Offspring Study reported that a high intake of phyto-estrogens in post-menopausal women is associated with a favorable metabolic cardiovascular risk profile. ${ }^{47,48}$ The beneficial effects of phyto-estrogens on CVDs, however, still need to be confirmed.

Bones

Some observational epidemiological studies reported that soy proteins and phyto-estrogens are beneficial for bone mass in post-menopausal women. ${ }^{49,50}$ Only a few randomized trials have been conducted on this issue, but recent trials seem to confirm that phyto-estrogens are effective against post-menopausal bone loss. ${ }^{51}$ However, the optimal dosage and the component responsible for the favorable effects are still unclear.

\section{Cognitive Function}

The effects of phyto-estrogens on the central nervous system in humans are poorly understood. Scattered reports suggest a beneficial effect of phyto-estrogens on memory, but the evidence on this issue is insufficient.

Breast Cancer

To date, 13 studies have been performed to assess the direct relationship between the individual dietary intake of soy products and the risk of breast cancer; however, none has reported statistically significant breastcancer reductions. Recent data indicate that surrogate markers of breast cancer risk — such as mammographic breast density-are not altered by phyto-estrogens, ${ }^{52}$ supporting the view that this class of compounds may act differently from standard hormonal therapies on the breast.

\section{Endometrial Cancer}

Reports on the effect of phyto-estrogens on endometrial cancer are limited. In Hawaii's multi-ethnic population, soy intake has been related to reduced endometrial-cancer risk..$^{53}$ Similar data have been found in non-Asian women in San Francisco. ${ }^{54}$

\section{Raloxifene}

Raloxifene is a non-steroidal selective estrogen receptor modulator (SERM). This compound induces estrogenic or anti-estrogenic actions depending on the tissue. Raloxifene is not effective on vasomotor symptoms, which can even be worsened during raloxifene administration, therefore making it an unsuitable agent for the treatment of symptomatic menopausal women.

\section{Cardiovascular System}

While previous trials suggested potential reduction of cardiovascular events in post-menopausal women receiving raloxifene, ${ }^{55}$ the recent publication of the Raloxifene Use for The Heart trial has instead shown no reduction of cardiovascular events. ${ }^{56}$ However, this compound is active in vascular cells where, in general, it behaves like an estrogen, possibly inducing protective effects. ${ }^{57,58}$

\section{Bones}

Raloxifene acts as a powerful estrogen on the bone, where it prevents bone loss and provides an effective treatment for osteoporosis. ${ }^{59}$

Breast Cancer

Recent evidence from the large Study of Tamoxifen and Raloxifene trial indicates that raloxifene administration to post-menopausal women results in a clinically relevant reduction of breast-cancer risk that is comparable to that achieved with tamoxifen. ${ }^{60}$

Newer SERMs with partially different characteristics are currently under 
development by the pharmaceutical industry and many of these compounds are in advanced clinical development.

\section{Conclusions}

While all the available therapies for early post-menopausal symptoms or for the prevention of the consequences of long-term estrogen deprivation have specific risk/benefit ratios, clinical selection is the key to maximizing the advantage for each patient. Overall, the safety profile of hormonal preparations is extremely reassuring, and the big claims of carcinogenetic actions of these drugs are not justified.
1. The Writing Group for the Women's Health Initiative Investigators, Risks and benefits of estrogen plus progestin in healthy postmenopausal women: principal results from the Women's Health Initiative randomized controlled trial, JAMA 2002;288:321-33.

2. Simoncini T, Genazzani AR, Timing is everything, Gynecol Endocrinol, 2007;23:1-4.

3. Herrington DM, Reboussin DM, Brosnihan KB, et al., Effects of estrogen replacement on the progression of coronary-artery atherosclerosis, N Engl J Med, 2000;343:522-9.

4. Rossouw JE, Prentice RL, Manson JE, et al., Postmenopausal hormone therapy and risk of cardiovascular disease by age and years since menopause, JAMA, 2007;297:1465-77.

5. Ettinger $B$, Pressman A, Sklarin $P$, et al., Associations between low levels of serum estradiol, bone density, and fractures among elderly women: the study of osteoporotic fractures, J Clin Endocrinol Metab, 1998:83:2239-43.

6. Lindsay R, Gallagher JC, Kleerekoper M, Pickar JH, Effect of lower doses of conjugated equine estrogens with and without medroxyprogesterone acetate on bone in early postmenopausal women, JAMA, 2002;287:2668-76.

7. Lees $B$, Stevenson JC, The prevention of osteoporosis using sequential low-dose hormone replacement therapy with estradiol17 beta and dydrogesterone, Osteoporos Int, 2001;12:251-8.

8. Stevenson JC, Teter P, Lees B, 17beta-estradiol (1 $\mathrm{mg} /$ day) continuously combined with dydrogesterone $(5,10$ or $20 \mathrm{mg} /$ day $)$ increases bone mineral density in postmenopausal women, Maturitas, 2001;38:197-203.

9. The Women's Health Initiative Steering Committee, Effects of conjugated equine estrogen in postmenopausal women with hysterectomy: The Women's Health Initiative Randomized Controlled Trial, JAMA, 2004;291:1701-12.

10. Stevenson JC, Justification for the use of HRT in the long-term prevention of osteoporosis, Maturitas, 2005;51:113-26.

11. Pike CJ, Estrogen modulates neuronal $\mathrm{BCl}-\mathrm{xL}$ expression and betaamyloid-induced apoptosis: relevance to Alzheimer's disease, J Neurochem, 1999;72:1552-63.

12. Henderson VW, Benke KS, Green RC, et al., Postmenopausal hormone therapy and Alzheimer's disease risk: interaction with age, I Neurol Neurosurg Psychiatry, 2005;76:103-5.

13. Shumaker $S A$, Legault $C$, Kuller $L$, et al., Conjugated equine estrogens and incidence of probable dementia and mild cognitive impairment in postmenopausal women: Women's Health Initiative Memory Study, JAMA, 2004;291:2947-58.

14. Resnick SM, Henderson VW, Hormone therapy and risk of Alzheimer's disease: a critical time, JAMA, 2002;288:2170-2.

15. Prest SJ, May FE, Westley BR, The estrogen-regulated protein, TFF1, stimulates migration of human breast cancer cells, Faseb J, 2002;16:592-4.

16. Collaborative Group on Hormonal Factors in Breast Cancer, Breast cancer and hormone replacement therapy: collaborative reanalysis of data from 51 epidemiological studies of 52,705 women with breast cancer and 108,411 women without breast cancer, Lancet, 1997:350:1047-59.

17. Chlebowski RT, Hendrix SL, Langer RD, Influence of estrogen plus progestin on breast cancer and mammography in healthy postmenopausal women: the Women's Health Initiative Randomized Trial, JAMA, 2003;289:3243-53.

18. Chen WY, Manson JE, Hankinson SE, Unopposed estrogen therapy and the risk of invasive breast cancer, Arch Intern Med, 2006;166:1027-32.

19. Fournier A, Berrino F, Riboli $E$, Breast cancer risk in relation to different types of hormone replacement therapy in the E3N-EPIC cohort, Int J Cancer, 2005;114:448-54.

20. Gambacciani M, Monteleone P, Sacco A, Genazzani AR, Hormone replacement therapy and endometrial, ovarian and colorectal cancer, Best Pract Res Clin Endocrinol Metab, 2003;17:139-47.

21. Persson I, Adami HO, Bergkvist L, Risk of endometrial cancer after treatment with oestrogens alone or in conjunction with progestogens: results of a prospective study, BMJ, 1989;298:147-51.

22. Weiderpass E, Adami HO, Baron JA, et al., Risk of endometrial cancer following estrogen replacement with and without progestins, J Natl Cancer Inst, 1999;91:1131-7.

23. Towler BP, Irwig L, Glasziou P, et al., Screening for colorectal cancer using the faecal occult blood test, hemoccult, Cochrane Database Syst Rev, 2000:CD001216.

24. Grodstein F, Newcomb PA, Stampfer MJ, Postmenopausal hormone therapy and the risk of colorectal cancer: a review and metaanalysis, Am J Med, 1999;106:574-82.

25. Smith CL, O'Malley BW, Coregulator function: a key to understanding tissue specificity of selective receptor modulators, Endocr Rev, 2004;25:45-71.

26. Hammar M, Christau S, Nathorst-Boos J, et al., A double-blind, randomised trial comparing the effects of tibolone and continuous combined hormone replacement therapy in postmenopausal women with menopausal symptoms, Br J Obstet Gynaecol, 1998;105:904-11.

27. Huber J, et al., Effects of tibolone and continuous combined hormone replacement therapy on bleeding rates, quality of life and tolerability in postmenopausal women, BJOG, 2002;109:886-93.

28. Baracat EC, Barbosa IC, Giordano MG, et al., A randomized, openlabel study of conjugated equine estrogens plus medroxyprogesterone acetate versus tibolone: effects on symptom control, bleeding pattern, lipid profile and tolerability, Climacteric, 2002;5:60-69.

29. Godsland IF, Effects of postmenopausal hormone replacement therapy on lipid, lipoprotein, and apolipoprotein (a) concentrations: analysis of studies published from 1974-2000 Fertil Steril, 2001;75:898-915.

30. Roux C, Pelissier C, Fechtenbaum J, et al., Randomized, doublemasked, 2-year comparison of tibolone with 17beta-estradiol and norethindrone acetate in preventing postmenopausal bone loss, Osteoporos Int, 2002;13:241-8.

31. Lippuner K, Haenggi W, Birkhaeuser MH, et al., Prevention of postmenopausal bone loss using tibolone or conventional peroral or transdermal hormone replacement therapy with 17betaestradiol and dydrogesterone, J Bone Miner Res, 1997;12:806-12.

32. Rymer J, Robinson J, Fogelman I, Ten years of treatment with tibolone $2.5 \mathrm{mg}$ daily: effects on bone loss in postmenopausal women, Climacteric, 2002;5:390-98.

33. Beral V, Breast cancer and hormone-replacement therapy in the Million Women Study, Lancet, 2003;362:419-27.

34. Valdivia I, Campodonico I, Tapia A, et al., Effects of tibolone and continuous combined hormone therapy on mammographic breast density and breast histochemical markers in postmenopausal women, Fertil Steril, 2004;81:617-23.

35. Wender MC, Edelweiss MI, Campos LS, et al., Endometrial assessment in women using tibolone or placebo: 1-year randomized trial and 2-year observational study, Menopause, 2004;11:423-9.

36. Kenemans $P$, Speroff $L$, Tibolone: clinical recommendations and practical guidelines, a report of the International Tibolone Consensus Group, Maturitas, 2005;51:21-8.

37. Axelson M, Sjovall J, Gustafsson BE, Setchell KD, Soya - a dietary source of the non-steroidal oestrogen equol in man and animals, Endocrinol, 1984;102:49-56.

38. Dalais FS, Rice GE, Wahlqvist ML, et al., Effects of dietary phytoestrogens in postmenopausal women, Climacteric, 1998;1:124-9.

39. St Germain A, Peterson CT, Robinson JG, Alekel DL, Isoflavone-rich or isoflavone-poor soy protein does not reduce menopausal symptoms during 24 weeks of treatment, Menopause, 2001;8:17-26.

40. Scambia G, Mango D, Signorile PG, et al., Clinical effects of a standardized soy extract in postmenopausal women: a pilot study,
Menopause, 2000;7:105-11.

41. Washburn S, Burke GL, Morgan T, Anthony M, Effect of soy protein supplementation on serum lipoproteins, blood pressure, and menopausal symptoms in perimenopausal women, Menopause, 1999;6:7-13.

42. Upmalis DH, Lobo R, Bradley L, et al., Vasomotor symptom relief by soy isoflavone extract tablets in postmenopausal women: a multicenter, double-blind, randomized, placebo-controlled study, Menopause, 2000;7:236-42.

43. Knight DC, Howes JB, Eden JA, Howes LG, Effects on menopausal symptoms and acceptability of isoflavone-containing soy powder dietary supplementation, Climacteric, 2001;4:13-18.

44. Kotsopoulos D, Dalais FS, Liang YL, et al., The effects of soy protein containing phytoestrogens on menopausal symptoms in postmenopausal women, Climacteric, 2000;3:161-7.

45. Tice JA, Ettinger B, Ensrud K, et al., Phytoestrogen supplements for the treatment of hot flashes: the Isoflavone Clover Extract (ICE) Study: a randomized controlled trial, JAMA, 2003;290:207-14.

46. Simoncini T, Fornari L, Mannella P, et al., Activation of nitric oxide synthesis in human endothelial cells by red clover extracts, Menopause, 2005;12:69-77.

47. Anderson JW, Johnstone BM, Cook-Newell ME, Meta-analysis of the effects of soy protein intake on serum lipids, $N$ Engl I Med, 1995; 333:276-82

48. de Kleijn MJ, van der Schouw YT, Wilson PW, et al., Dietary intake of phytoestrogens is associated with a favorable metabolic cardiovascular risk profile in postmenopausal U.S. women: the Framingham study, J Nutr, 2002;132:276-82.

49. Horiuchi T, Onouchi T, Takahashi M, et al., Effect of soy protein on bone metabolism in postmenopausal Japanese women, Osteoporos Int, 2000;11:721-4.

50. Mei J, Yeung SS, Kung AW, High dietary phytoestrogen intake is associated with higher bone mineral density in postmenopausal but not premenopausal women, I Clin Endocrinol Metab, 2001;86:5217-21.

51. Atkinson C, Compston JE, Day NE, et al., The effects of phytoestrogen isoflavones on bone density in women: a doubleblind, randomized, placebo-controlled trial, Am I Clin Nutr, 2004; 79:326-33.

52. Atkinson C, Warren RM, Sala E, et al., Red clover-derived isoflavones and mammographic breast density: a double-blind, randomized, placebo-controlled trial, Breast Cancer Res, 2004:6:R170-79.

53. Goodman MT, Wilkens LR, Hankin JH, et al., Association of soy and fiber consumption with the risk of endometrial cancer, Am J Epidemiol, 1997;146:294-306.

54. Usui T, Pharmaceutical prospects of phytoestrogens, Endocr J, 2006:53:7-20

55. Barrett-Connor E, Grady D, Sashegyi A, et al., Raloxifene and cardiovascular events in osteoporotic postmenopausal women: four-year results from the MORE (Multiple Outcomes of Raloxifene Evaluation) randomized trial, JAMA, 2002;287:847-57.

56. Barrett-Connor $E$, Mosca $L$, Collins $P$, et al., Effects of raloxifene on cardiovascular events and breast cancer in postmenopausal women, N Engl J Med, 2006;355:125-37.

57. Simoncini T, Genazzani AR, Raloxifene acutely stimulates nitric oxide release from human endothelial cells via an activation of endothelial nitric oxide synthase, J Clin Endocrinol Metab, 2000:85:2966-9.

58. Simoncini T, Genazzani AR, Liao JK, Nongenomic mechanisms of endothelial nitric oxide synthase activation by the selective estrogen receptor modulator raloxifene, Circulation, 2002;105:1368-73.

59. Delmas PD, et al., Effects of raloxifene on bone mineral density, serum cholesterol concentrations, and uterine endometrium in postmenopausal women, N Engl J Med, 1997;337:1641-7.

60. Vogel VG, Costantino JP, Wickerham DL, et al., Effects of tamoxifen vs raloxifene on the risk of developing invasive breast cancer and other disease outcomes: the NSABP Study of Tamoxifen and Raloxifene (STAR) P-2 trial, JAMA, 2006;295:2727-41. 